\title{
p13 overexpression in pancreatic $\beta$-cells ameliorates type 2 diabetes in high-fat-fed mice
}

Shintaro Higashi ${ }^{1,2,13}$, Kazuhiko Katagi ${ }^{1,13}$, Norihito Shintani ${ }^{1,3,13}$, Kazuya Ikeda ${ }^{1,2}$, Yukihiko Sugimoto $^{4,5}$, Soken Tsuchiya ${ }^{4,5}$, Naoki Inoue ${ }^{1}$, Shota Tanaka ${ }^{1}$, Mai Koumoto ${ }^{1}$, Atsushi Kasai ${ }^{1}$, Takanobu Nakazawa ${ }^{1,6}$, Atsuko Hayata-Takano ${ }^{7}$, Ken-ichi Hamagami ${ }^{1}$, Shuhei Tomimoto ${ }^{1}$, Takuya Yoshida $^{8}$, Tadayasu Ohkubo $^{8}$, Kazuki Nagayasu ${ }^{1,6}$, Yukio Ago ${ }^{9}$, Yusuke Onaka ${ }^{1,2,9}$, Ryota Hashimoto $^{7,10}$, Atsushi Ichikawa ${ }^{4,11}$, Akemichi Baba ${ }^{1,12^{*}}$, Hitoshi Hashimoto ${ }^{1,6,7^{*}}$

${ }^{1}$ Laboratory of Molecular Neuropharmacology, Graduate School of Pharmaceutical Sciences, Osaka University, 1-6 Yamadaoka, Suita, Osaka 565-0871, Japan

${ }^{2}$ Research Fellow of the Japan Society for the Promotion of Science

${ }^{3}$ Department of Biology, University of Padua, 35121 Padua, Italy

${ }^{4}$ Department of Physiological Chemistry, Faculty of Pharmaceutical Sciences, Kyoto University, Kyoto, Japan

${ }^{5}$ Department of Pharmaceutical Biochemistry, Faculty of Medical and Pharmaceutical Sciences, Kumamoto University, Kumamoto 862-0973, Japan

${ }^{6}$ iPS Cell-based Research Project on Brain Neuropharmacology and Toxicology, Graduate School of Pharmaceutical Sciences, Osaka University, 1-6 Yamadaoka, Suita, Osaka 565-0871, Japan

${ }^{7}$ Molecular Research Center for Children’s Mental Development, United Graduate School of Child Development, Osaka University, Kanazawa University, Hamamatsu University School of Medicine, Chiba University and University of Fukui, 2-2 Yamadaoka, Suita, Osaka 565-0871, Japan

${ }^{8}$ Laboratory of Biophysical Chemistry, Graduate School of Pharmaceutical Sciences, Osaka University, 1-6 Yamadaoka, Suita, Osaka 565-0871, Japan

${ }^{9}$ Laboratory of Medicinal Pharmacology, Graduate School of Pharmaceutical Sciences, Osaka University, 1-6 Yamadaoka, Suita, Osaka 565-0871, Japan

${ }^{10}$ Department of Psychiatry, Graduate School of Medicine, Osaka University, 1-6 Yamadaoka, 
Suita, Osaka 565-0871, Japan

${ }^{11}$ Institute for Biosciences, Mukogawa Women’s University, Kyu-ban cho, Koshien, Nishinomiya, Hyogo 663-8179, Japan

${ }^{12}$ Faculty of Pharmaceutical Sciences, Hyogo University of Health Science, 1-3-6

Minatojima, Chuo-ku, Kobe, Hyogo 650-8530, Japan

${ }^{13}$ These authors equally contributed to this work.

*Corresponding Authors:

Akemichi Baba, PhD. (Co-corresponding)

Hyogo University of Health Science, 1-3-6 Minatojima, Chuo-ku, Kobe, Hyogo 650-8530, Japan

Tel.: +81 78304 3080; Fax: +81 78304 2780; E-mail: akbaba@huhs.ac.jp

Hitoshi Hashimoto, PhD. (Co-corresponding author who communicates with the editorial office)

Laboratory of Molecular Neuropharmacology, Graduate School of Pharmaceutical Sciences, Osaka University, 1-6 Yamadaoka, Suita, Osaka 565-0871, Japan

Tel.: +81 66879 8180; Fax: +81 66879 8184; E-mail: hasimoto@phs.osaka-u.ac.jp

\section{Abbreviations}

4-HNE, 4-hydroxynonenal;

HFD, high-fat diet

OGTT, oral glucose tolerance test;

p13-Tg mice, transgenic mice overexpressing p13 in pancreatic $\beta$-cells;

PACAP, pituitary adenylate cyclase-activating polypeptide;

STD, standard diet 


\begin{abstract}
We examined the pancreatic function of p13 encoded by 1110001J03Rik, whose expression is decreased in pancreatic islets in high-fat-fed diabetic mice, by generating transgenic mice overexpressing p13 (p13-Tg) in pancreatic $\beta$-cells. p13-Tg mice showed normal basal glucose metabolism; however, under high-fat feeding, these animals showed augmented glucose-induced first-phase and total insulin secretion, improved glucose disposal, greater islet area and increased mitotic insulin-positive cells. In addition, high-fat diet-induced 4-hydroxynonenal immunoreactivity, a reliable marker and causative agent of lipid peroxidative stress, was significantly decreased in p13-Tg mouse islets. These results indicate that p13 is a novel pancreatic factor exerting multiple beneficial effects against type 2 diabetes.
\end{abstract}

\title{
Keywords:
}

1110001J03Rik; glucose-induced insulin secretion; high-fat diet; lipid peroxidative stress; PACAP; type 2 diabetes 


\section{Introduction}

The major pathophysiologic abnormalities of type 2 diabetes include insulin resistance and decreased $\beta$-cell function, the latter of which includes loss of $\beta$-cell mass and $\beta$-cell dysfunction [1-4]. Pituitary adenylate cyclase-activating polypeptide (PACAP) [5] belongs to the same family as glucagon-like peptide-1, and these two peptides share insulinotropic and insulin-sensitizing properties [6-10]. Yada et al. have shown that PACAP serves as an endogenous amplifier of glucose-induced insulin secretion, and long-term administration of PACAP improves hyperglycemia in Goto-Kakizaki (GK) rats, an animal model of type 2 diabetes [6, 11]. In addition, Ahrén et al. have shown that dipeptidyl peptidase-4 (DPP-4) inhibition-mediated insulin responses involve not only glucagon-like peptide-1 but also PACAP38 [12]. We previously reported that overexpression of PACAP in pancreatic $\beta$-cells enhances insulin secretion, ameliorates streptozotocin-induced diabetes [13], normalizes hyperplasia of pancreatic islets and attenuates hyperinsulinemia in mouse models of type 2 diabetes [14-17]. In addition, after culture under high glucose or palmitate conditions, Nakata et al. showed that increases in glucose-induced insulin secretion and first-phase cytosolic $\mathrm{Ca}^{2+}$ concentrations were severely impaired in the islets of PACAP-deficient mice [18]. These results suggest that PACAP plays not only insulinotropic but also multiple anti-diabetic roles.

To understand the mechanisms responsible for these observations, we conducted a gene chip analysis in the hyperplastic pancreatic islets of agouti yellow diabetic mice. We identified a cDNA from the functionally uncharacterized RIKEN 1110001J03 gene (1110001J03Rik) along with genes of predicted function, such as regenerating gene III $\beta$ (RegIII $\beta$ ), which was down-regulated in the pancreas of transgenic mice overexpressing PACAP in pancreatic $\beta$-cells [19].

In the present study, we examined the in vivo role of the 1110001J03Rik gene product (NP_079639), which consists of 113 amino acids $(12.68 \mathrm{kDa})$ and is hereafter referred to as p13. We examined the expression of p13 in the pancreatic islets of type 2 diabetic mice and generated transgenic mice overexpressing p13 in pancreatic $\beta$-cells under control of the human insulin promoter (p13-Tg). 


\section{Materials and Methods}

\section{Animals}

All animal care and handling procedures were performed according to the Guidelines for the Care and Use of Laboratory Animals of the Japanese Pharmacological Society. The Animal Care and Use Committee of the Graduate School of Pharmaceutical Sciences, Osaka University approved all procedures. All efforts were made to minimize the number of animals used.

Mice were housed under a 12-h light-dark cycle (lights on at 8:00 a.m.) and fed a standard diet containing $11.8 \mathrm{kcal} \%$ fat (STD; DC-8, CLEA, Osaka, Japan) and water ad libitum. For the high-fat diet (HFD) feeding study, mice were singly housed and fed rodent food containing $60 \mathrm{kcal} \%$ fat (D12492, Research Diet, New Brunswick, NJ, USA) or standard diet (STD) as a control for 12 weeks starting at 8 weeks of age.

The mouse p13 cDNA was amplified using reverse transcription PCR with the following primers: sense 5’-CGG AGC TCC CGC CAT GGC GGC CCT GGG GTC CCC G-3’ and antisense 5’-CGG AGC TCT TAC GGC TCC CAG CCC TTC CCT C-3’. The resulting PCR product of $0.34 \mathrm{~kb}$ was subcloned into the EcoRI site of the pIns-1 vector ([20], a gift from Dr. Jun-ichi Miyazaki, Osaka University Graduate School of Medicine, Japan). In this vector, the p13 cDNA is driven by the $1.9 \mathrm{~kb}$ human insulin promoter. After the accuracy of construction was confirmed by DNA sequencing, the resultant transgene was microinjected into the pronuclei of fertilized eggs of (C57BL/6 × DBA/2) F1 (BDF1) hybrid mice using standard procedures. Transgenic mice were identified by PCR genotyping from tail DNA using the following exon-specific primers: sense 5'-AAG GCT TTC CGA GCA CAT CGG-3' (exon 1) and antisense 5'-CAG CCC TTC CCT CCA GGC TGA C-3' (exon 2). Among several transgenic founders, we established one line, which was crossed to wild-type C57BL/6N mice (Shimizu Laboratories Supplies, Kyoto, Japan) for more than 6 generations. Only male mice were used for this study, and non-transgenic littermates of p13-Tg mice were used as controls. 


\section{Histochemical analyses}

After mice were deeply anesthetized, they were transcardially perfused with $4 \%$ paraformaldehyde in phosphate-buffered saline. The pancreas was removed, embedded in paraffin, and sectioned at $5-\mu \mathrm{m}$ thickness, and the sections were deparaffinized for hematoxylin-eosin or immunohistochemical staining. After blocking for $20 \mathrm{~min}$ in $2 \%$ goat or horse serum, the sections were incubated with rabbit anti-p13 antibody (1:250; produced by Takara Bio Inc., Kyoto, Japan), guinea pig anti-insulin antibody (1:500, Dako, Glostrup, Denmark), mouse anti-4-hydroxynonenal (4-HNE) modified protein antibody $(12.5 \mu \mathrm{g} / \mathrm{ml}$, Nikken SEIL, Tokyo, Japan) or rabbit anti-Ki-67 antibody (1:200, Abcam, Cambridge, England) overnight at $4^{\circ} \mathrm{C}$. The primary antibodies were detected using Alexa Fluor 488- or 594-conjugated secondary antibodies (1:1,000, Invitrogen, Carlsbad, CA, USA) of the corresponding species, FITC-conjugated anti-guinea pig IgG antibody (1:1,000, Invitrogen), or biotinylated secondary antibodies of the corresponding species followed by Vectastain ABC kit for diaminobenzidine staining (Vector Laboratories, CA, USA). The relative intensity of the diaminobenzidine signal (immunoreactivity), pancreas area, islet number and islet area were analyzed using ImageJ software (NIH Image, Bethesda, MD, USA).

\section{Measurements of body weight, food intake, plasma glucose and insulin levels, and oral glucose tolerance test (OGTT)}

The procedures were performed as previously described [14-17]. Food intake was measured by weighing the residual food in the tray. Body weight and food intake were measured every week from 8 to 20 weeks of age. For measurements of plasma glucose and insulin levels, blood was sampled from the tail vein at 8, 11, 14, 17 and 20 weeks of age. The glucose and insulin levels were determined using the Glucose CII-test (Wako Pure Chemicals, Osaka, Japan) and an Ultra-Sensitive Mouse Insulin ELISA kit (Morinaga Institute of Biological Science, Inc., Yokohama, Japan), respectively, according to the manufacturers' protocols. For OGTT, mice were orally injected with $2 \mathrm{~g} / \mathrm{kg}$ body weight of glucose after an 8-hour fast. Blood was sampled from the tail vein just prior to time 0 and at 15, 30, 60, 90 and 120 min after the injection. 


\section{Data analysis and statistics}

Statistical evaluation was performed using Statview software (SAS Institute Japan Ltd., Tokyo, Japan). The statistical significance of differences was assessed using two-way or repeated-measures two-way ANOVA followed by the Tukey-Kramer test or Student's unpaired t-test, where applicable. Differences with $P<0.05$ were considered significant.

\section{Results}

\section{p13 expression is decreased in islets of HFD-fed mice compared with STD-fed mice}

p13 expression levels were examined immunohistochemically in pancreatic islets and were compared between mice fed with STD or HFD for 12 weeks. In the pancreas of mice fed with STD, p13 immunoreactivity was clearly observed in the islets (Supplementary Fig. 1A). In mice fed with HFD, striking hyperplasia of pancreatic islets was observed, as expected, and their p13 expression levels were decreased to 66\% compared with the islets of STD-fed mice (Supplementary Fig. 1B, C). These results suggested that pancreatic p13 was expressed mainly in islets in the pancreas, and p13 expression was down-regulated in type 2 diabetic islets.

\section{Generation of p13-Tg mice}

Given that p13 expression was down-regulated in the type 2 diabetic pancreas, we next addressed whether the type 2 diabetic phenotypes could be rescued by transgenic overexpression of p13 in mice. We therefore generated p13-Tg mice, which express human insulin promoter-driven p13 in pancreatic $\beta$-cells (Fig. 1A) and backcrossed them onto the C57BL/6 background for at least 6 generations. Through the course of backcrossing, p13-Tg mice were indistinguishable from their non-transgenic littermates with respect to growth, fertility, and apparent behavior (data not shown). Immunohistochemical analysis revealed increased p13 immunoreactivity in pancreatic islets in both STD- and HFD-fed p13-Tg mice compared with non-transgenic mice (Fig. 1B-D). 


\section{Normal age-dependent changes in metabolic parameters but enhanced glucose-induced insulin secretion and glucose disposal in HFD-fed p13-Tg mice}

In both STD- and HFD-fed states, body weight, food intake, basal plasma glucose and insulin levels were not different between p13-Tg and non-transgenic mice from 8 to 20 weeks of age (Fig. 2). We then conducted the OGTT in p13-Tg and non-transgenic mice fed with HFD. Although basal (fasting) plasma glucose was not different between p13-Tg and non-transgenic mice, the glucose-induced increase in plasma glucose levels was significantly attenuated in p13-Tg mice at 120 min after glucose administration (Fig. 3A). The area under the curve of glucose levels for 90-120 min was also significantly decreased in p13-Tg mice compared with non-transgenic mice (Fig. 3B). In addition, the glucose-induced increase in plasma insulin levels was significantly enhanced in p13-Tg mice (Fig. 3C). Glucose-induced first-phase insulin secretion, as determined by the area under the curve of insulin levels above basal for the first $30 \mathrm{~min}$ after glucose administration and an increasing ratio of insulin levels at 15 min above $0 \mathrm{~min}$, was significantly increased in p13-Tg mice compared with non-transgenic mice (Fig. 3D). All these measures in the OGTT did not significantly differ between p13-Tg and non-transgenic mice when fed with STD (data not shown).

\section{Enhanced proliferation of pancreatic $\beta$-cells in HFD-fed p13-Tg mice}

To address possible mechanisms underpinning the improved first-phase insulin response in HFD-fed p13-Tg mice, we conducted histomorphometric analysis of pancreatic islets 2 weeks after the end of the HFD feeding study (Fig. 4). Mean islet area, islet density (islet number per pancreatic area), and islet mass were not significantly different between p13-Tg and non-transgenic mice when fed a STD. However, the HFD-induced increase in mean islet area was more prominent in p13-Tg mice compared with non-transgenic mice (Fig. 4A, F). We then analyzed intra-islet mitotic cells by immunocytochemistry for the Ki-67 antigen and observed that the ratio of Ki-67-positive cells among insulin-positive cells was significantly increased in p13-Tg mice compared with non-transgenic mice when fed with HFD. However, this ratio did not differ between p13-Tg and non-transgenic mice when the animals were fed 
with STD (Fig. 4D, G).

\section{Attenuated lipid peroxidation in islets of HFD-fed p13-Tg mice}

To further address the in vivo role of p13, we examined oxidative stress levels in islets by immunohistochemical staining for 4-HNE, a reliable lipid peroxidation marker and causative agent of oxidative stress. Although 4-HNE immunoreactivity in islets did not differ between p13-Tg and non-transgenic mice when fed with STD, HFD-increased 4-HNE immunoreactivity was significantly attenuated in p13-Tg mice compared with non-transgenic mice (Fig. 4E and H).

\section{Discussion}

In the present study, we aimed to address the functions of p13 protein in the pancreatic islet and showed that p13 expression was decreased in the islets of HFD-fed mice. Transgenic overexpression of p13 in pancreatic $\beta$-cells in mice caused no obvious changes in glucose metabolism; however, p13 improved glucose-induced first-phase insulin secretion, mildly increased glucose disposal during the OGTT and islet area, and augmented mitotic islet insulin-positive cells under high-fat feeding. Furthermore, p13 overexpression attenuated HFD-induced islet lipid peroxidation as assessed by 4-HNE levels. These results suggest that a hitherto functionally uncharacterized p13 exerts multiple beneficial effects against HFD-induced type 2 diabetes.

The observation that age-dependent changes in body weight, food intake, plasma glucose and insulin levels were not changed in p13-Tg mice fed either with STD or HFD at the basal state compared with non-transgenic littermates suggests that p13 is not critically involved in basal insulin secretion or glucose disposal. By contrast, under HFD feeding, glucose-stimulated insulin secretion in the OGTT was augmented in p13-Tg mice. First-phase insulin secretion, also termed the acute insulin response, and subsequent second-phase secretion are known to be impaired or absent in type 2 diabetes patients [21, 22]. Therefore, it is plausible that maintaining p13 expression levels could contribute to preservation of the 
insulin secretory response.

This possibility is supported by the present observations that HFD-fed p13-Tg mice showed significantly increased mitotic insulin-positive cells. Indeed, several studies in humans and rodents have postulated that $\beta$-cells have a capacity for increased proliferation in response to increased insulin demands, which might be useful for identifying new therapeutic strategies to preserve $\beta$-cell functional mass [23]. Thus, the present study may contribute to better understanding of the protective and adaptive mechanisms for increasing functional $\beta$-cells.

Oxidative stress can inactivate key islet transcription factors, producing stunned $\beta$-cells that temporarily lose glucose sensitivity [4, 24], and rescuing this defect is a key therapeutic target [25]. The present observations that HFD-induced increases in 4-HNE levels are considerably attenuated in p13-Tg mice may suggest a mechanism for the improved first-phase insulin secretion and increased mitotic insulin-positive cells, further supporting the protective roles of p13 in type 2 diabetes.

In the present study, we could not determine the mechanism by which p13 exerts the observed actions in HFD-fed mice because the cellular functions of p13 have not yet been elucidated. We are currently addressing this issue in another study.

In conclusion, the present study shows that p13, a novel protein expressed in pancreatic $\beta$-cells, exerts multiple beneficial and protective effects against HFD-induced type 2 diabetes. It is expected that this study will help to better understand and identify potential therapeutic targets for type 2 diabetes.

\section{Acknowledgments}

We thank Professor Jun-ichi Miyazaki at Osaka University Medical School for the pIns-1 vector and valuable advice. This work was supported in part by the Japan Society for the Promotion of Science (JSPS) Grants-in-Aid for Scientific Research, KAKENHI; Seeds Innovation Project from the Japan Science and Technology Agency (JST) (to AB); Project MEET, Osaka University Graduate School of Medicine (to NS); Mitsubishi Tanabe Pharma 
Corporation (to NS); JSPS Funding Program for Next Generation World-Leading Researchers, Grant number LS081 (to HH); JSPS Program for Advancing Strategic International Networks to Accelerate the Circulation of Talented Researchers, Grant No. S2603 (to HH); JSPS Research Fellowships for Young Scientists (to SH, KI, and YO); and grants for research from the Uehara Memorial Foundation, Japan (to NS and HH). 


\section{Figure legends}

Fig. 1. Generation of p13-Tg mice that express human insulin promoter-driven $p 13$ in pancreatic $\boldsymbol{\beta}$-cells. A, Schematic representation of the fusion gene used to generate p13-Tg mice. The fusion gene consists of the 1.9 kb-human insulin promoter (hatched box), fragments of the rabbit $\beta$-globin gene (open boxes), and $0.34 \mathrm{~kb}$ of the p13 cDNA (black box). The lines indicate the second intron and 3'-flanking region of the rabbit $\beta$-globin gene. $\mathbf{B}-\mathbf{C}$, Representative images of p13 immunostaining showing overexpression of p13 in pancreatic islets of p13-Tg mice fed with STD (C) or HFD (D) compared with non-transgenic (non-Tg) littermates fed with STD (B). Scale bar, $200 \mu \mathrm{m}$.

Fig. 2. Age-dependent changes in metabolic parameters in p13-Tg mice fed with HFD or STD. Body weight (A), food intake (B), plasma glucose (C) and insulin levels (D) were determined at the indicated ages in p13-Tg mice and non-transgenic (non-Tg) littermates fed with HFD or STD. Values are expressed as the means \pm SEM ( $=9-15$ per group). $* * P<$ 0.01, repeated two-way ANOVA.

Fig. 3. The OGTT showing enhanced glucose-induced first-phase insulin secretion in HFD-fed p13-Tg mice. After 8 hours of fasting, glucose (2 g/kg body weight) was orally administered to 22-week-old p13-Tg mice and non-transgenic (non-Tg) littermates fed with HFD, and time-dependent changes in plasma glucose (A) and insulin levels (C) were measured ( $n=11-15$ per group). The area under the curve (AUC) of glucose levels for 0-90 min and 90-120 min (B), insulin levels above basal for the first $30 \mathrm{~min}$ and the increasing ratio of insulin levels at $15 \mathrm{~min}$ above $0 \mathrm{~min}(\mathbf{D})$ were calculated. Values are expressed as the means \pm SEM. ${ }^{\#} P<0.05$ compared with non-transgenic mice at the same time point, two-way ANOVA followed by Student's t-test; $* P<0.05$, $* * P<0.01$, Student's t-test.

Fig. 4. Enhanced proliferation of pancreatic $\beta$-cells and attenuated 4-HNE-immunoreactivity in islets of HFD-fed p13-Tg mice. A-E, The mean islet area (A), 
the number of islets per square millimeter (islet density; B), the calculated total islet mass (C), the percentage of Ki-67-positive cells among insulin-positive cells (D), and 4-HNE-immunoreactive intensity (E) in 22-week-old p13-Tg mice and non-transgenic (non-Tg) littermates fed with HFD or STD. F-H, Representative images of hematoxylineosin staining (F), immunostaining for Ki-67 (green), insulin (red) and Hoechst 33258 (blue; G), and 4-HNE immunostaining $(\mathbf{H})$ of pancreatic sections. Inset, magnified image of boxed area. Values are expressed as the means \pm SEM ( $\mathrm{n}=7-11$ per group). ${ }^{*} \mathrm{P}<0.05,{ }^{* *} P<0.01$, two-way ANOVA followed by Tukey-Kramer test. Scale bars, $200 \mu \mathrm{m}$ in F, $50 \mu \mathrm{m}$ in $\mathbf{G}$ and $100 \mu \mathrm{m}$ in $\mathbf{H}$. 


\section{References}

[1] J.E. Shaw, R.A. Sicree, P.Z. Zimmet, Global estimates of the prevalence of diabetes for 2010 and 2030, Diabetes Res. Clin. Pract. 87 (2010) 4-14.

[2] Y.M. Farag, M.R. Gaballa, Diabesity: an overview of a rising epidemic, Nephrol. Dial. Transplant. 26 (2011) 28-35.

[3] F.M. Ashcroft, P. Rorsman, Diabetes mellitus and the beta cell: the last ten years, Cell 148 (2012) 1160-1171.

[4] P.A. Halban, K.S. Polonsky, D.W. Bowden, et al., beta-cell failure in type 2 diabetes: postulated mechanisms and prospects for prevention and treatment, J. Clin. Endocrinol. Metab. 99 (2014) 1983-1992.

[5] A. Miyata, A. Arimura, R.R. Dahl, et al., Isolation of a novel 38 residue-hypothalamic polypeptide which stimulates adenylate cyclase in pituitary cells, Biochem. Biophys. Res. Commun. 164 (1989) 567-574.

[6] T. Yada, M. Sakurada, K. Ihida, et al., Pituitary adenylate cyclase activating polypeptide is an extraordinarily potent intra-pancreatic regulator of insulin secretion from islet beta-cells, J. Biol. Chem. 269 (1994) 1290-1293.

[7] K. Filipsson, M. Kvist-Reimer, B. Ahren, The neuropeptide pituitary adenylate cyclase-activating polypeptide and islet function, Diabetes 50 (2001) 1959-1969.

[8] B. Ahren, Role of pituitary adenylate cyclase-activating polypeptide in the pancreatic endocrine system, Ann. N. Y. Acad. Sci. 1144 (2008) 28-35.

[9] D. Vaudry, A. Falluel-Morel, S. Bourgault, et al., Pituitary adenylate cyclase-activating polypeptide and its receptors: 20 years after the discovery, Pharmacol. Rev. 61 (2009) 283-357.

[10] B. Omar, B. Ahren, Pleiotropic mechanisms for the glucose-lowering action of DPP-4 inhibitors, Diabetes 63 (2014) 2196-2202.

[11] T. Yada, M. Sakurada, K. Filipsson, et al., Intraperitoneal PACAP administration decreases blood glucose in GK rats, and in normal and high fat diet mice, Ann. N. Y. Acad. Sci. 921 (2000) 259-263.

[12] B. Ahren, T.E. Hughes, Inhibition of dipeptidyl peptidase-4 augments insulin secretion in 
response to exogenously administered glucagon-like peptide-1, glucose-dependent insulinotropic polypeptide, pituitary adenylate cyclase-activating polypeptide, and gastrin-releasing peptide in mice, Endocrinology 146 (2005) 2055-2059.

[13] K. Yamamoto, H. Hashimoto, S. Tomimoto, et al., Overexpression of PACAP in transgenic mouse pancreatic beta-cells enhances insulin secretion and ameliorates streptozotocin-induced diabetes, Diabetes 52 (2003) 1155-1162.

[14] S. Tomimoto, H. Hashimoto, N. Shintani, et al., Overexpression of pituitary adenylate cyclase-activating polypeptide in islets inhibits hyperinsulinemia and islet hyperplasia in agouti yellow mice, J. Pharmacol. Exp. Ther. 309 (2004) 796-803.

[15] Y. Sakurai, N. Shintani, A. Hayata, et al., Trophic effects of PACAP on pancreatic islets: a mini-review, J. Mol. Neurosci. 43 (2011) 3-7.

[16] Y. Sakurai, H. Inoue, N. Shintani, et al., Compensatory recovery of blood glucose levels in $\mathrm{KKA}(\mathrm{y})$ mice fed a high-fat diet: insulin-sparing effects of PACAP overexpression in beta cells, J. Mol. Neurosci. 48 (2012) 647-653.

[17] H. Inoue, N. Shintani, Y. Sakurai, et al., PACAP Inhibits beta-cell Mass Expansion in a Mouse Model of Type II Diabetes: Persistent Suppressive Effects on Islet Density, Front. Endocrinol. (Lausanne) 4 (2013) 27.

[18] M. Nakata, N. Shintani, H. Hashimoto, et al., Intra-islet PACAP protects pancreatic beta-cells against glucotoxicity and lipotoxicity, J. Mol. Neurosci. 42 (2010) 404-410.

[19] K. Hamagami, Y. Sakurai, N. Shintani, et al., Over-expression of pancreatic pituitary adenylate cyclase-activating polypeptide (PACAP) aggravates cerulein-induced acute pancreatitis in mice, J. Pharmacol. Sci. 110 (2009) 451-458.

[20] J. Miyazaki, K. Araki, E. Yamato, et al., Establishment of a pancreatic beta cell line that retains glucose-inducible insulin secretion: special reference to expression of glucose transporter isoforms, Endocrinology 127 (1990) 126-132.

[21] S. Seino, Cell signalling in insulin secretion: the molecular targets of ATP, cAMP and sulfonylurea, Diabetologia 55 (2012) 2096-2108.

[22] E. Ferrannini, A. Mari, beta-Cell function in type 2 diabetes, Metabolism 63 (2014) 1217-1227. 
[23] K.Y. Jung, K.M. Kim, S. Lim, Therapeutic Approaches for Preserving or Restoring Pancreatic beta-Cell Function and Mass, Diabetes Metab. J. 38 (2014) 426-436.

[24] S. Guo, C. Dai, M. Guo, et al., Inactivation of specific beta cell transcription factors in type 2 diabetes, J. Clin. Invest. 123 (2013) 3305-3316.

[25] E. Ferrannini, The stunned beta cell: a brief history, Cell Metab. 11 (2010) 349-352. 


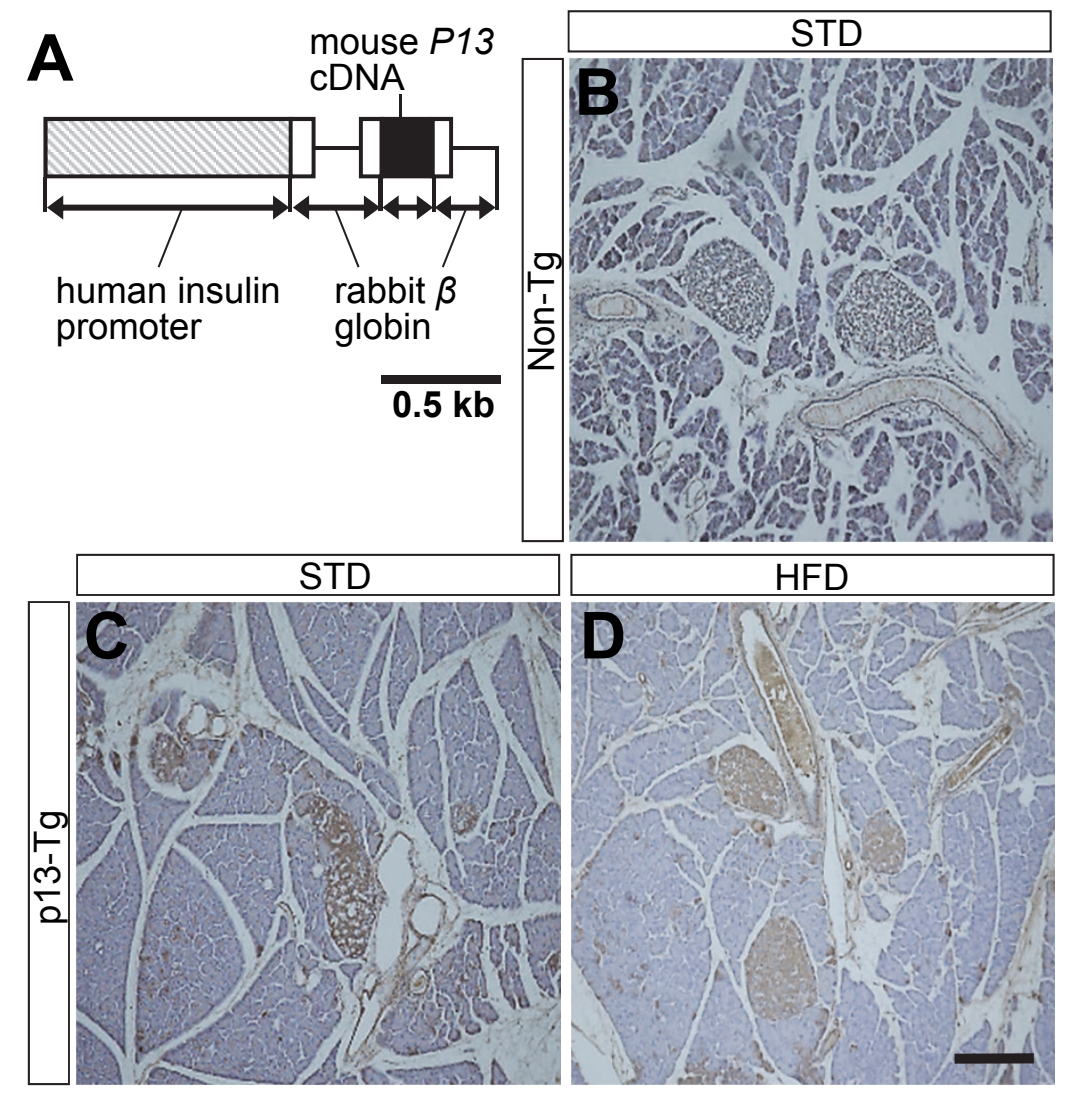

Fig. 1. Higashi et al. 

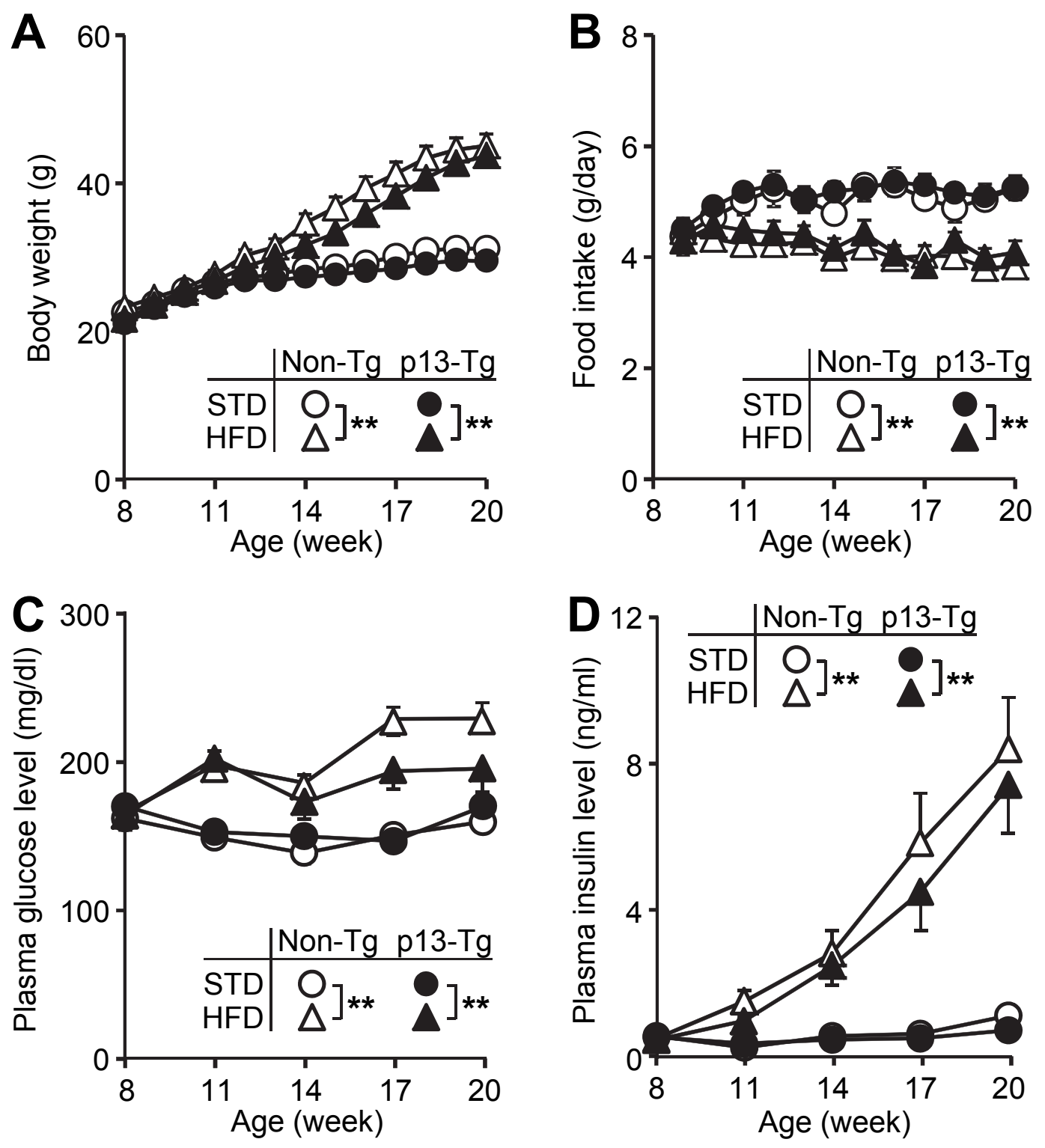

Fig. 2. Higashi et al. 

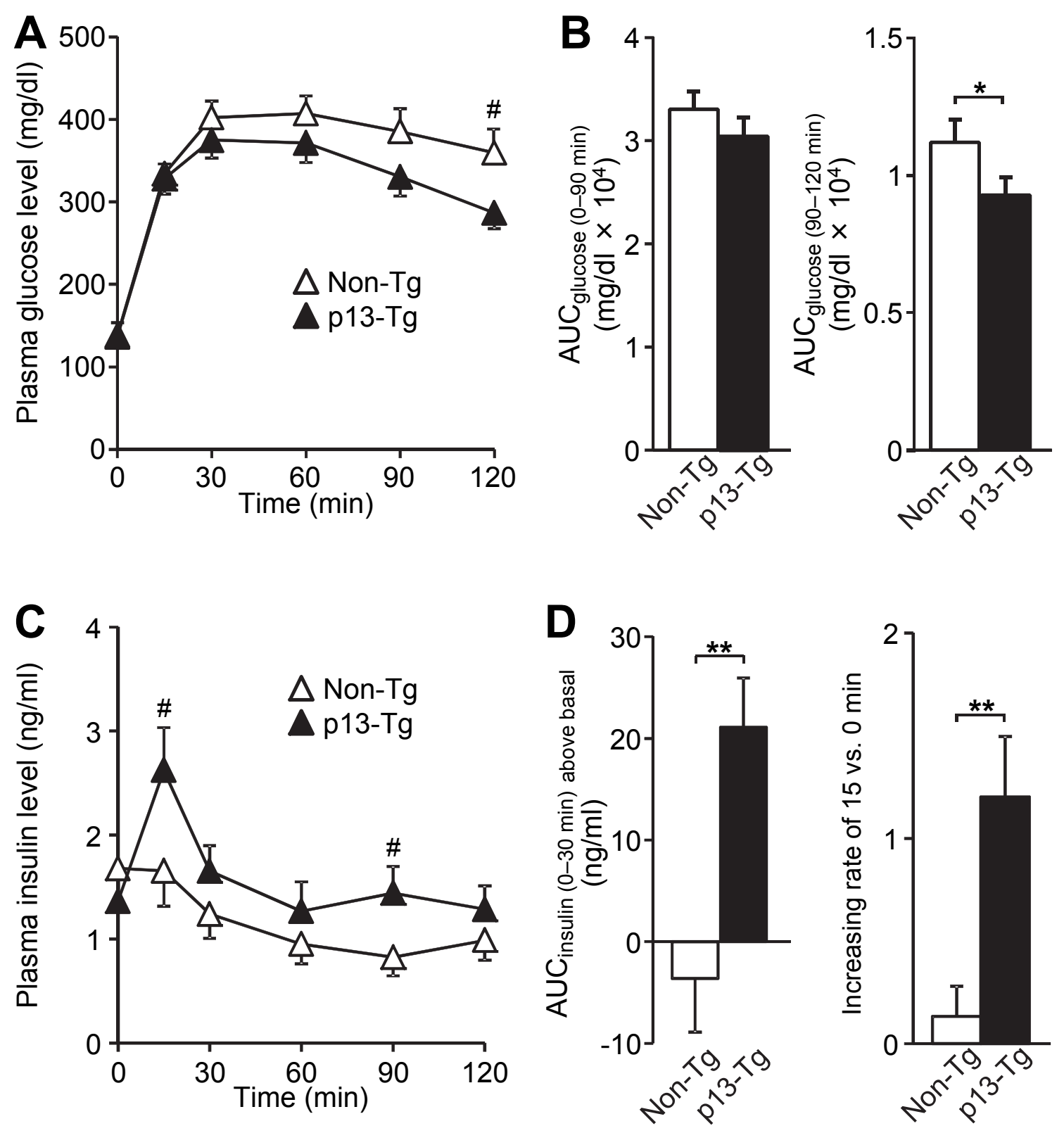

Fig. 3. Higashi et al. 

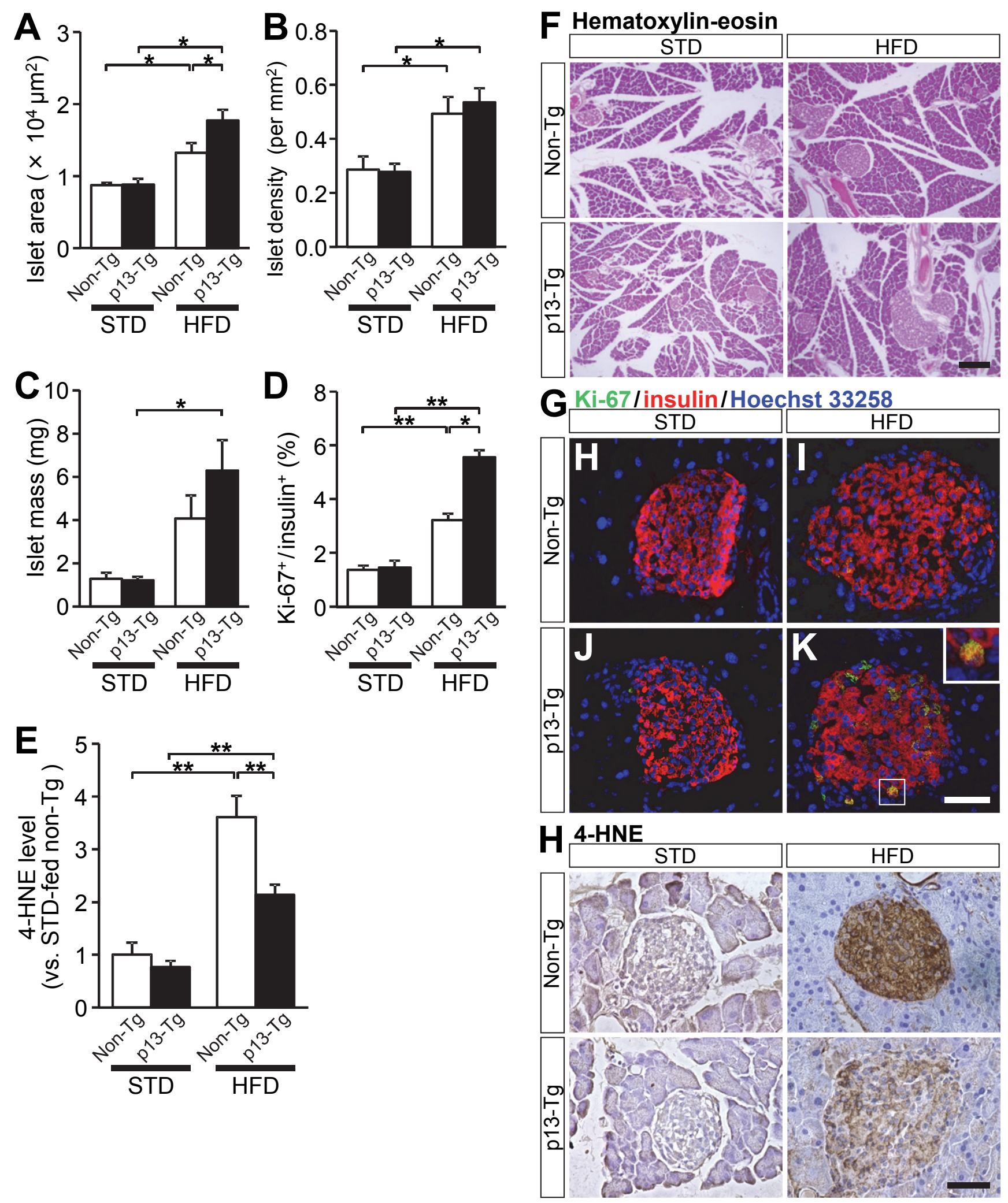

Fig. 4. Higashi et al. 